\title{
Therapeutic importance of garlic supplementation in the management of nonalcoholic fatty liver disease: A systematic review of preclinical studies
}

\begin{abstract}
Background: Non-alcoholic fatty liver disease (NAFLD) is considered as the most widely recognized incessant liver problem. It is accepted to be the hepatic segment of the metabolic disorder. Numerous treatment approaches have been proposed, the most encouraging of which are those with way of life interventions. This preclinical review was performed due to lack of articles on human subject during the literature search. This systematic review will help to explore basic concepts about the mechanisms by which garlic supplementation suppresses NAFLD.
\end{abstract}

Objective: The aim of this systematic review was to evaluate the therapeutic importance of garlic supplementation in NAFLD.

Methods: The PUBMED, Cochrane central library and Google Scholar were searched. The search items were limited to only randomize controlled preclinical trials with English language irrespective of species, age, gender and time. In vitro and human studies were excluded. Key outcomes were a change in serum transaminase, body weight and liver histochemistry.

Results: Initial searching retrieved 13,341 peer-reviewed articles and abstracts. Eight studies met all inclusion criteria. The review included a total of 361 animal models. Following delivery of garlic extracts, a decrease in serum transaminases, a reduction of high fat diet induced hepatic lipid and body weight was observed. Additional outcomes like a reduction of pro-inflammatory cytokines and oxidative stress markers and an enhancement of antioxidative enzymes were seen in some of the studies.

Conclusion: The decrease in a key biochemical marker of liver damage following treatment with garlic extracts, as well as improvements in liver histochemistry, suggests that these agents may be effective alternatives for managing NAFLD.

Registration: CRD42018087907

Keywords: garlic supplementation, NAFLD and garlic supplementation, oxidative stress, garlic or steatosis
Volume 8 Issue 3 - 2020

\begin{abstract}
Getnet Fetene,' Sintayehu Ambachew, ${ }^{2}$ Alebachew Fasil, ${ }^{2}$ Molla Abebe $^{2}$

'University of Gondar Comprehensive Specialized Hospital, University of Gondar, Ethiopia

${ }^{2}$ Department of Clinical Chemistry, School of Biomedical and Laboratory Sciences, College of Medicine and Health Sciences, University of Gondar, Ethiopia
\end{abstract}

\begin{abstract}
Correspondence: Getnet Fetene Teshome, University of Gondar Comprehensive Specialized Hospital, University of Gondar, Gondar, Ethiopia, Tel +2519-1809-8902, Email getnetfetene44@gmail.com
\end{abstract}

Received: April 16, 2020 | Published: May 04, 2020

\begin{abstract}
Abbreviations: ACC, Acyl CoA Carboxylase; ALT, Alanine Aminotransferase; AMPK, Adenine Monophosphate activated protein kinase; ApoB, Apoprotein B; AST, Aspartate Aminotransferase; CCl4, carbon tetrachloride, CPT-1, Carnitine Palmitoyl Transferase-1; CYP2E1, Cytochrome P450 2E1; DADS, Diallyl Disulfide; ERK, extracellular regulatory Kinase; FAS, fatty acid synthase; GEO, garlic essential oil; HCC, hepatocellular carcinoma; HFD, high fat diet; GSH, glutathione; GRx, glutathione reductase; GST, glutathione synthase; IL-1 $\beta$, Interleukin-1 $\beta$; IR, insulin resistance, LXR- $\alpha$, liver $\mathrm{X}$ receptor- $\alpha$; LKB1, liver Kinase B1, MAPK; mitogen activated protein Kinase; MDA, Malondialdehyde; NAFLD, nonalcoholic fatty liver disease; NASH, nonalcoholic Steatohepatitis; PI3K, phosphoinositide 3-kinase; PNPLA3, patatin-like phospholipase domain-containing3; ROS, reactive oxygen species; S6K1, S6 Kinase 1; SAMC, S-allylmercaptocysteine; SREBP1c, Sterol regulatory element binding protein-1c; T2DM, type 2 diabetes mellitus; TC, total cholesterol; TG, triglyceride; TNF- $\alpha$, Tumor Necrosis Factor- $\alpha$; TM6SF2,Transmembrane 6 super family member2; VLDL, very low density lipoprotein
\end{abstract}

\section{Introduction}

Nonalcoholic fatty liver disease is one of the most common liver disease in the developed world. ${ }^{\top}$ The term NAFLD portrays a wide range of liver illnesses extending from simple steatosis(fat in the liver) to its more serious frame nonalcoholic steatohepatitis (NASH) with hepatic damage, inflammation and frequently with fibrosis. ${ }^{2}$ NAFLD is characterized as fat collection in the liver surpassing $5 \%$ to $10 \%$ by weight ${ }^{3}$ or it can be described as hepatic steatosis not caused by overabundance utilization of liquor $(>20 \mathrm{~g} /$ day in ladies, $>30 \mathrm{~g} /$ day in men), infections(for example hepatitis virus), autoimmunity and utilization of hepatotoxic medications or other compounds. ${ }^{4} \mathrm{NASH}$ is characterized by steatosis, necro-inflammation and cytopathic changes and it will progress to cirrhosis. ${ }^{5} \mathrm{NASH}$, but not NAFLD, carries the risk of disease progression and development of complications such as cirrhosis and hepatocellular carcinoma (HCC). ${ }^{6}$

Nonalcoholic fatty liver disease can be ordered in to four classes in light of histological findings. Class 1 constitutes simple steatosis without liver damage though class 2 is steatosis with lobular inflammation. Class 3 and class 4 require the presence of ballooned (swelled) hepatocytes and Mallory's hyaline or fibrosis respectively. 
Based on this categorization, class 3 and class 4 are similar and might be considered as a single component to NASH. ${ }^{7}$

Insulin resistance (IR), obesity and type 2 diabetes mellitus (T2DM) are associated with NAFLD. The headliners in the pathophysiology of NAFLD are lipid gathering, lipotoxicity and inflammation. ${ }^{6}$ Increased dietary admission, de-novo lipogenesis and convergence of free fatty acids (FFAs) are factors that lead to lipid aggregation in the liver NAFLD and T2DM share common features like IR and dyslipidemia. IR incites lipolysis in the adipose tissue which increases inundation of FFAs in to the liver. ${ }^{8}$ A connection amongst IR and NAFLD has been perceived for a long time, however, whether IR is an etiological factor of NAFLD or simply an accompanying feature of NAFLD continues to be debated. IR in liver and adipose tissue prompts lessened glucose uptake and hyperglycemia and the resultant increment in discharge of insulin favors triglyceride (TG) stockpiling in the liver.IR is also believed to play a role in the development of inflammatory changes and later fibrosis in relation to NAFLD. Insulin resistant conditions have pro-inflammatory effects observed in insulin sensitive tissues such as adipose tissue and liver. When abundance fat is deposited in the liver, this may enhance inflammation locally as unsaturated fats in high amounts have pro-inflammatory impacts in their own particular right. ${ }^{9,10}$

Despite the fact that there is an awesome enthusiasm for distinguishing NAFLD, the natural history of this condition stays unclear. In general, some patients seem to be characterized by an indolent course, whereas others develop advanced necroinflammation and liver fibrosis progressing to an end stage liver disease. ${ }^{11}$ Patients with simple steatosis generally have a benign long term prognosis without severe histological progression, whereas patients with NASH reveals much more aggressive natural history with development of cirrhosis in up to $26 \%$ of the patients. ${ }^{5}$

The presence of hepatocytes damage and death is the hallmark of NASH. The pathogenic mechanism that drive NAFLD movement to NASH and NASH to fibrosis are unpredictable/complex. Multiple hits resulted from oxidative stress, cytokines and hormonal deregulation are suggested. These hits overwhelm homeostatic mechanisms within fatty hepatocytes which in turn induce cellular damage and apoptosis. ${ }^{12} \mathrm{An}$ increased number of apoptotic hepatocytes in patients with NASH was observed ${ }^{13}$ and demonstrated that the magnitude of apoptosis positively correlated with disease severity. ${ }^{14}$

In spite of high prevalence and the consequent public health burden, it is surprising that there are no FDA approved therapies for NAFLD. Currently, vitamin E and pioglitazone are recommended treatments with great safety concern in selected patients..$^{15}$ Even though there is much effort, there are challenges in the management of NAFLD. Lifestyle interventions are patient centered and maintaining the desired outcome is ideal. Dietary and exercise therapies are challenging because of lack of behavioral knowledge and motivation. To bring the desired result, patients always need motivation from health care providers. ${ }^{16}$ In addition some safety issues are raised with long term uses of some drugs. For instance, pioglitazone and vitamin $\mathrm{E}$ are reported with side effects of hemorrhagic stroke, risk of bladder cancer, postmenopausal bone loss and prostate cancer. ${ }^{17,18}$ The objective of this review was to assess the significance of garlic extracts on the management of NAFLD.

Recent studies has concentrated on garlic extracts containing organo sulfur compounds which can be either water soluble or oil soluble. Water soluble garlic extracts include aged black
garlic(ABG), S-allylcysteine, S-allyl mercapto cysteine(SAMC) and S-methylcysteineand oil soluble sulfur containing compounds include, garlic essential oil(GEO), diallyl sulfide, diallyl disulfide(DADS), diallyltrisulfideand ajoene. . $^{19,20}$

In this paper, we overviewed the significance of garlic in the management of NAFLD. Garlic (Allium sativum) is a member of a lily family. It is a widely consumed customary food and has various medicinal properties. Garlic had been utilized as a remedy for diseases in ancient Egyptian about 10,000 years ago. Recent evidences have revealed a variety of its biological activities, including anticarcenogenic, antimutagenic, antithrombosis and cardioprotection. Garlic has likewise been recommended to have antifungal, antimicrobial, hypoglycaemic, hypolipidaemic, antihypertensive and hepatoprotective properties. ${ }^{21-23}$ Our review will something in the body of scientific knowledge. Since there is no approved pharmacotherapy to treat NAFLD and constraint of RCTs, it will provide clues for researchers and any stakeholders.

\section{Method}

Techniques of the analysis and eligibility criteria were specified prior to the literature search and documented in a protocol registered with Prospero (CRD42018087907).This systematic review was conducted in accordance with the preferred reporting item for systematic review and meta-analysis (PRISMA) guidelines ${ }^{24}$ and recommendations described in the protocol format for the preparation, registration and publication of systematic reviews of animal intervention studies ${ }^{25}$ including independent execution of search strategy and bias assessment.

\section{Literature search}

The PUBMED and Cochrane central databases were searched from conception through February 8, 2018. In order to incorporate all available papers, we used additional search engines such as Google Scholar. The search terms were 'Garlic supplementation', 'NAFLD and Garlic supplementation', oxidative stress', Garlic or steatosis'

\section{Inclusion criteria}

We reviewed only studies on animal models because of limited studies on human. The search items were limited to only randomize preclinical controlled trials of in vivo animal studies of any kind, age and gender, which were published with English language. The studies with a diagnosis of HFD induced steatosis, NASH or fibrosis made on validated biochemical and histological evidences were included. Animal studies investigating the effect of garlic on HFD induced liver injury and LFC by analyzing the levels of ALT and/or AST and histologic evaluation of the LFC were eligible for this review. Furthermore, animal studies evaluating the effect of garlic on lobular inflammation, hepatocellular ballooning and fibrosis as outcome measurement were included.

We used two phases in screening, namely initial screening based on title and abstract, followed by full-text screening of the eligible articles for final inclusion. In each phase, two observers were independently assessed each article. Any disagreement between them over the eligibility of particular studies was resolved through discussion with a third reviewer.

\section{Exclusion criteria}

Studies excluded from this review include review papers and 
studies conducted are not an in vivo animal studies, without control group, not about HFD induced NAFLD and without the outcome of interest.

\section{Quality assessment}

The quality of each included study was assessed by using the SYRCLE's risk of bias tool for animal studies. ${ }^{26} \mathrm{We}$ obtained information from each trial as recommended by SYRCLE's risk of bias tool for animal studies. These are:

a. The objective of the intervention

b. Method of recruitment of participants

c. The inclusion and exclusion criteria

d. If well designed protocol was followed

e. Whether or not ethical approval had been granted

f. Whether or not presence of any funding sources

g. The statistical methods used

h. Methods of randomization

i. Incomplete outcome data reporting, any selective reporting and other sources of bias. ${ }^{26}$

In addition, we assessed the methods of assessing outcome measures, the reliability and validity of the outcome measures, the methods of housing and follow-up, the timing of the outcome measurement and any adverse events. The primary outcome measurement of the treatment effects were histologic evaluation, levels of liver enzymes, improvement in inflammation, hepatocellular and ballooning, change in fibrosis, oxidative stress markers and inflammatory markers.

\section{Data abstraction}

Two reviewers were independently extracted data from each article. We tried to extract numerical data from tables, text or figures. Experimental groups were HFD induced animal models whereas control groups were animal models receiving normal or laboratory diet. Data was extracted regardless of weight, sex, age, dose administered and follow up period. English language articles without restriction of time of publication were abstracted.

Liver enzymes and histochemistry of liver were primary outcomes of this review. The secondary outcomes of this review were metabolic components, inflammation and oxidative stress markers. Data were abstracted with regard to the following characteristics of the included studies; author, publication year, sample size, animal model, intervention strategy (regimen, dosage, route, and duration), control (normal diet or other) and outcomes. The data type is dichotomous that is either improved or aggravated as compared to control or negative control. The result was summarized with table. Any discrepancy was resolved by discussion and consensus with a third reviewer.

\section{Strategy for data synthesis}

We did not do meta-analysis because of the range of different outcomes measured across the small number of existing trials and the possibility of different measurement approach for a single outcome. Therefore, the scope of this work is systematic review. We provided a narrative synthesis of the findings from the included studies, structured around the type of intervention, target population characteristics, type of outcome, doses of intervention and follow up time.

\section{Result}

We identified and selected eligible studies included in this review through the database searches, as sown in Figure 1. Overall 13,341 records were identified, out of which 13,320 were removed due to duplication. The remaining 21 publications were screened for eligibility by reading their titles and abstracts and 11 papers were excluded. Publications excluded were either human studies, interventions other than garlic or its derivatives, review papers, trials not assessing NAFLD markers as a primary outcome and non-English articles. A total of 10 papers were selected for further assessment after title and abstract screening. Two papers were excluded after reading the full print of each paper for the following reasons; one was experimental study on human cell and the other was a randomized control trial of human participant.

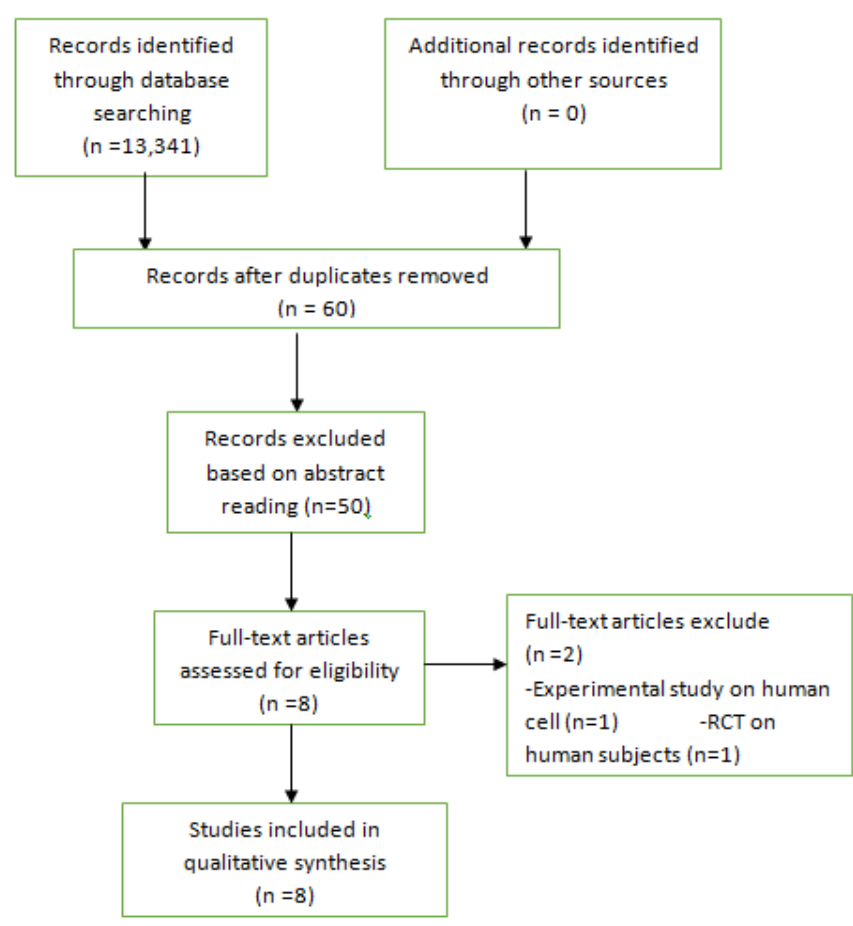

Figure I Study flow diagram.

\section{Trial characteristics}

Overall, 361 animal models were participated in 8 preclinical studies with durations ranging from 4 weeks to 3 months. Of the 8 studies that met the inclusion criteria, two assessed the effect of SAMC, ${ }^{27,28}$ one assessed the effect of Aged Black Garlic Extract, ${ }^{29}$ two evaluated the effect of aqueous garlic extract, ${ }^{30,31}$ one compared garlic essential oil (GEO) and its major organosulfur component DADS, ${ }^{32}$ one investigated ajoene, a stable by-product of garlic ${ }^{33}$ and the remaining one evaluated fresh crushed garlic ${ }^{34}$ on diet induced NAFLD. All of the included preclinical studies used rodents, mice or rats, except one study done on rabbits by Arhan et al. ${ }^{30}$ Two studies ${ }^{27,28}$ were done exclusively on female rats whereas two $0^{31,33}$ underwent on only male mice. The protocols of the included trials were very diverse, in which the amount of garlic extracts ranged from $1.5 \mathrm{ml} / \mathrm{kg} /$ day to $500 \mathrm{mg} / \mathrm{kg} / \mathrm{day}$. The routes of administration of the laboratory diet and garlic were either through intravenous ${ }^{27,28}$ or oral. ${ }^{29-34}$ All studies utilized liver histochemistry as outcome measurement and most of the studies used liver enzymes mainly ALT level as outcome measurement in addition to histochemistry. 


\section{Quality assessment evidence}

Common limitations of the studies included were inadequate randomization, inadequate allocation concealment, lack of personnel blinding, inadequate description of baseline characteristics and inadequate information how experimental animals were housed or caged (Table 1).

Treatment of garlic extracts have been reported to reduce body weight. After administration of mice with 25,50 , or $100 \mathrm{mg} / \mathrm{kg} /$ day GEO and 10 or $20 \mathrm{mg} / \mathrm{kg} /$ day DADS for 12 weeks, Lai et al., ${ }^{32}$ found final body weights of $41.05 \mathrm{gm}, 32.95 \mathrm{gm}, 29.25 \mathrm{gm}, 43.92 \mathrm{gmand} 36.38$ gm respectively as compared to the HFD only group $(50.29 \mathrm{gm})$ and controls group(31.23gm). Han et al., ${ }^{33}$ and Shin et al., ${ }^{29}$ demonstrated a similar result after administration of $30 \mathrm{~g} / \mathrm{k} /$ day of ajoene for 4 weeks and 100 or $200 \mathrm{gm} / \mathrm{kg} /$ day aged black garlic(ABG) respectively.

Table I SYRCLE's risk of bias tool for animal studies checklist carried out on the 7 eligible preclinical studies

\begin{tabular}{|c|c|c|c|c|c|c|c|c|c|}
\hline \multirow{2}{*}{ Quality criteria } & & \multicolumn{8}{|l|}{ Studies } \\
\hline & & Arhan $\mathbf{M}^{30}$ & Han $Y C^{33}$ & Xiao J $\mathbf{J}^{27}$ & Xiao J ${ }^{28}$ & Lai $Y^{32}$ & Shin $\mathrm{HJ}^{29}$ & Qamar $A^{34}$ & El-Din ${ }^{31}$ \\
\hline \multirow{3}{*}{ Selection bias } & Sequence generation & $U$ & $\mathrm{U}$ & $U$ & $U$ & $U$ & $U$ & $U$ & $U$ \\
\hline & Baseline characteristics & $U$ & $\mathrm{U}$ & $U$ & $U$ & $\mathrm{U}$ & $U$ & + & - \\
\hline & Allocation concealment & $\cup$ & $\cup$ & $\cup$ & $\cup$ & $\mathrm{U}$ & 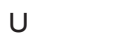 & $\cup$ & $\cup$ \\
\hline \multirow{2}{*}{ Performance bias } & Random housing & $\mathrm{U}$ & $U$ & $U$ & $\cup$ & + & $U$ & + & + \\
\hline & Blinding & $U$ & $\mathrm{U}$ & $\mathrm{U}$ & $U$ & + & $U$ & - & - \\
\hline \multirow[t]{2}{*}{ Detection bias } & $\begin{array}{l}\text { Random outcome } \\
\text { assessment }\end{array}$ & + & + & + & + & + & + & + & + \\
\hline & Blinding & $U$ & $U$ & $U$ & $U$ & $U$ & $U$ & $U$ & $U$ \\
\hline Attrition bias & Incomplete outcome data & + & + & + & + & + & + & + & + \\
\hline Reporting bias & $\begin{array}{l}\text { Selective outcome } \\
\text { reporting }\end{array}$ & + & + & + & + & + & + & + & + \\
\hline \multicolumn{2}{|c|}{ Other sources of bias } & + & + & + & + & + & + & + & + \\
\hline
\end{tabular}

$U$ denoted unclear, + denoted the requirement is fulfilled, - denoted the requirement is not fulfilled.

Han et al., ${ }^{33}$ observed a significant reduction of ALT level after administration of $30 \mathrm{gm} / \mathrm{kg} /$ day of ajoene for 4 weeks. Similarly Shin et al., ${ }^{29}$ reported a significant reduction of both serum ALT and AST levels after treatment with 100 or $200 \mathrm{gm} / \mathrm{kg} /$ day. After 12 weeks of HFD feeding, Lai et al., ${ }^{32}$ found a significant elevation of serum ALT and AST level by 5.5 and 2 fold, respectively. However, GEO and DADS significantly lowered ALT and ASTin dose dependent manner. Xiao et al., ${ }^{27}$ found a marked reduction of serum ALT level upon intraperitoneal administration of $200 \mathrm{gm} / \mathrm{kg} / \mathrm{day}$ SAMC for 8 weeks. Insignificant improvement of ALT and AST levels were found by Arhanet al., ${ }^{30}$ from administration of $1.5 \mathrm{ml} / \mathrm{kg} / \mathrm{day}$ for 3 months on rabbit model. El-Din et al., ${ }^{31}$ has demonstrated a significant effect in lowering HFD induced ALT,AST and ALP level after $500 \mathrm{mg} / \mathrm{Kg} /$ day garlic supplementation for 20 weeks.

Garlic was found to be effective in improvement of hepatic steatosis, reduction of pro-inflammatory and oxidative stress markers and increment of anti-oxidative stress markers. Lai et al., ${ }^{32}$ observed a significant reduction of LFC, malondialdehyde (MDA), TNF- $\alpha$, interleukin-1 $\beta($ IL-1 $\beta$ ), interleukin-6(IL-6), sterol regulatory element binding protein(SREPB-1c) and its targets fatty acid synthase(FAS) and acyl-CoA carboxylase (ACC) after treatment with GEO and DADS. In addition, glutathione(GSH), glutathione reductase (GRx), glutathione peroxidase (GPx), glutathione superoxide dismutase (GSOD), catalase and glutathione-S-synthase (GST) were markedly increased by treatment of GEO and DADS. ${ }^{32}$ Similarly, Xiao et al., ${ }^{27}$ Han et al., ${ }^{33}$ and El-Din et al., ${ }^{31}$ reported a significant attenuation of oxidative stress markers, pro-inflammatory cytokine and lipogenic gene expression and enhancement of antioxidative enzymes. ${ }^{27,33}$ Arhan et al., ${ }^{30}$ found improvement of steatosis, lobular inflammation, ballooning and fibrosis after administration of garlic. Treatment with garlic extract was also found to be effective in enhancing the expression of key autophagic markers and down regulating apoptotic factors (Table 2). ${ }^{28}$

\section{Discussion}

Regardless of the huge effort put in the prevention and treatment of NAFLD, there are few options to retard or even reverse the progression of this disease. Recently, several drugs including anti-obesity regimens, insulin sensitizers, antihyperlipidemics and antioxidants have been assessed for the treatment of NAFLD. However, a few of them brought very positive outcomes. To date, weight management with life style intervention is the most recognized therapeutic option to improve liver injury induced by NAFLD.${ }^{35}$ However, the significance of lifestyle intervention depends on patient's own efforts and sometimes this is typically hard to achieve and maintain. ${ }^{36}$ Treatment with metformin may be beneficial in promoting moderate weight loss and metabolic improvement. Treatment with pioglitazone has been recommended in improvement of all liver histological features. ${ }^{37}$ However, side effects related with the long-term use of these drugs including weight gain, congestive heart failure, cardiovascular morbidity, bone loss and increased risk for urinary bladder cancer put these drugs with great safety concern. ${ }^{38}$ Due to limited availability of 
pharmacotherapies, there are much research interests in this area ${ }^{35}$ and currently garlic extracts are under investigation.

Lai et al., ${ }^{32}$ observed a significant improvement of body weight as compared to HFD induced mice after administration of GEO and DADS for 12 weeks. Similar result was reported by Shin et al., ${ }^{29}$ and
Han et al., ${ }^{33}$ after treatment of HFD induced mice with ABG and ajoene for 4 and 8 weeks respectively. Likewise, El-Din et al., ${ }^{31}$ observed a significant reduction of body and liver weight after administration of rat models with garlic alone or combined with onion for 8 weeks. Weight loss was recommended for treatment of NAFLD by American Association for the Study of Liver Diseases. ${ }^{35}$

Table 2 Trials evaluating the role of garlic on diet induced nonalcoholic fatty liver disease

\begin{tabular}{|c|c|c|c|c|c|c|}
\hline Reference & $\begin{array}{l}\text { Sample } \\
\text { size }\end{array}$ & diagnosis & Intervention & Control & Fup & Key findings \\
\hline Arhan et al., ${ }^{30}$ & 31 rabbit & hepatic histology & $\begin{array}{l}\text { Cholesterol }(0.5 \mathrm{~g} / \mathrm{kg} / \text { day }) \text { for } 4 \\
\text { months } \\
7 \mathrm{killed=group} \mathrm{II} \\
7 \mathrm{ND} \text { for } 3 \mathrm{months}=\text { group III } \\
8 \mathrm{ND}+\text { garlic }(\mathrm{I} .5 \mathrm{ml} / \mathrm{kg} / \mathrm{day}) \text { for } \\
3 \text { months=group IV }\end{array}$ & ND & 3 mths & $\begin{array}{l}\text { Reduction of steatosis and } \\
\text { lobular inflammation in garlic } \\
\text { treated Lowered levels of the } \\
\text { liver cholesterol, TG and serum } \\
\text { cholesterol significantly in garlic } \\
\text { treated compared to group III } \\
\text { Insignificant for ALT and AST }\end{array}$ \\
\hline Han et al., ${ }^{33}$ & $32 M$ & $\begin{array}{l}\text { hepatic histology } \\
\text { and } \\
\text { serum ALT }\end{array}$ & $\begin{array}{l}\text { Oral HFD }(13,320 \% \text { of } \mathrm{kcal} \text { as fat }) \\
\text { HFD + vehicle, } \\
\text { HFD+ ajoene }(10 \mathrm{mg} / \mathrm{kg} / \text { day }) \text { and } \\
\text { HFD + ajoene }(30 \mathrm{mg} / \mathrm{kg} / \text { day }) \text {, } \\
\mathrm{n}=8 \text { each }\end{array}$ & ND & $8 w k s$ & $\begin{array}{l}\text { LFC at either } 10 \mathrm{mg} / \mathrm{kg} \text { or } 30 \mathrm{mg} / \\
\mathrm{kg}, \mathrm{ALT} \text { activity at } 30 \mathrm{mg} / \mathrm{kg} \text {, } \\
\text { Reduced body weight }\end{array}$ \\
\hline Xiao et al., ${ }^{27}$ & $28 \mathrm{~F}$ & $\begin{array}{l}\text { hepatic histology } \\
\text { and } \\
\text { serum ALT }\end{array}$ & $\begin{array}{l}\text { NAFLD group, } \\
\text { SAMC Rx group }(200 \mathrm{mg} / \mathrm{kg} \\
\text { Injection three times per week); } \\
\text { and } \\
\text { NAFLD and SAMC cotreatment } \\
\text { group }\end{array}$ & ND & $8 w k s$ & $\begin{array}{l}\text { SAMC Rx markedly reduced } \\
\text { hepatic injury(ALT),infn and } \\
\text { collagen, FFAs SREBPIc and up- } \\
\text { regulating adiponectin }\end{array}$ \\
\hline Xiao et al., ${ }^{28}$ & $28 \mathrm{~F}$ & $\begin{array}{l}\text { hepatic histology } \\
\text { and } \\
\text { serum ALT }\end{array}$ & $\begin{array}{l}\text { NAFLD group, } \\
\text { SAMC Rx group }(200 \mathrm{mg} / \mathrm{kg} \\
\text { Injection three times per week); } \\
\text { and } \\
\text { NAFLD and SAMC } \\
\text { cotreatment group }\end{array}$ & ND & $8 w k s$ & $\begin{array}{l}\text { Reduced apoptotic pathway, } \\
\text { upregulated autophagic pathway }\end{array}$ \\
\hline Lai et al., ${ }^{32}$ & 70 & $\begin{array}{l}\text { hepatic histology } \\
\text { and } \\
\text { serum ALT and AST }\end{array}$ & $\begin{array}{l}\text { Oral HFD(I3,320\% kcal from fat, } \\
\text { DI2492) } \\
\text { HFD } \\
\text { HFD+GEO }(25,50,100 \mathrm{mg} / \mathrm{kg}) \\
\text { HFD+DAS }(10,20 \mathrm{mg} / \mathrm{kg}\end{array}$ & $\begin{array}{l}\text { LFD }(13.5 \% \\
\text { kcal from fat, } \\
\text { Laboratory } \\
\text { Rodent Diet } \\
5001)\end{array}$ & $12 w k s$ & $\begin{array}{l}25,50 \text { and } 100 \mathrm{mg} / \mathrm{kg} \text { of GEO } \\
\text { reduced AST levels } \\
\text { by } 34.1,39.1 \text {, and } 46.4 \% \text {, } \\
\text { respectively and ALT by } 43.0 \text {, } \\
67.1 \text {, and } 84.2 \% \text {, } \\
\text { Reduced HFC, body weight } \\
\text { dose dependently }\end{array}$ \\
\hline El-Din et al., ${ }^{31}$ & $90 M$ & $\begin{array}{l}\text { Liver histology, } \\
\text { lipids, parameters } \\
\text { of oxidative stress, } \\
\text { TNF- } \alpha \text { and TGF- } \beta \\
\text { were measured }\end{array}$ & $\begin{array}{l}9 \text { groups; normal (I), NAFLD } \\
\text { induced with high fat diet (HFD; } \\
\text { II), NAFLD switched to regular } \\
\text { diet (RD; III), NAFLD-HFD or } \\
\text { NAFLD-RD treated with garlic (IV, } \\
\text { V), onion (VI,VII) or the } \\
\text { combined garlic+onion (VIII,IX) } \\
\text { garlic=500mg/Kg, onion= } 100 \mathrm{mg} / \mathrm{Kg}\end{array}$ & vehicle & $20 w k s$ & $\begin{array}{l}\text { Garlic decreased ALT, AST, leptin, } \\
\text { TC,TG,TNF- } \alpha \text {, TGF- } \beta \text { and } \\
\text { hepatic MDA } \\
\text { Garlic increased GSH, GR, } \\
\text { GPx, GST, SOD and serum } \\
\text { adiponectin. } \\
\text { But insignificant for ALP }\end{array}$ \\
\hline Shin et al., ${ }^{29}$ & 32 & $\begin{array}{l}\text { hepatic histology } \\
\text { and serum ALT,AST } \\
\text { and ALP }\end{array}$ & $\begin{array}{l}\text { Oral HFD=DI2492 } \\
\text { HFD+ vehicle } \\
\text { HFD+ ABG }(100 \mathrm{mg} / \mathrm{kg}) \\
\text { HFD + ABG }(200 \mathrm{mg} / \mathrm{kg})\end{array}$ & $C D=D \mid 2450 B)$ & $4 w k s$ & $\begin{array}{l}\text { Improved HFD-induced increase } \\
\text { in AST and ALT, but not ALP, and } \\
\text { lipid profiles was significantly } \\
\text { suppressed by ABG treatment } \\
\text { Body weight induced by HFD } \\
\text { was significantly reduced, but } \\
\text { not CCl and D-galactosamine } \\
\text { induced WT }\end{array}$ \\
\hline $\begin{array}{l}\text { Qamar et } \\
\text { al., }{ }^{34}\end{array}$ & 50 & hepatic histology & $\begin{array}{l}\text { SFD }=20 \mathrm{gm} / 100 \mathrm{gm} \text { of diet orally } \\
\text { UFD }=20 \mathrm{gm} / 100 \mathrm{gm} \text { orally } \\
\text { Butter+Garlic orally } \\
\text { Corn oil+Garlic orally }\end{array}$ & ND & $8 w k s$ & $\begin{array}{l}\text { Reduced deposition of fat in } \\
\text { butter-and-garlic treated } \\
\text { Group D and corn-oil-with- } \\
\text { garlic-fed Group E animals can }\end{array}$ \\
\hline
\end{tabular}

ALP, alkaline phosphatase; $A L T$, alanine aminotransferase; $A S T$, aspartate aminotransferase; $A B G$, aged black garlic; $C D$, control diet; $F$, female; FFAs, free fatty acids; fup, follow up period; GEO, garlic essential oil; HFD, high fat diet; LFC, liver fat content; LFD, low fat diet; mths, months; NAFLD, nonalcoholic fatty liver disease; ND, normal diet; Rx, treatment; SAMC, S-allylmercaptocysteine; SFD, saturated fat diet; TC, total cholesterol; TG, triglyceride; UFD, unsaturated fat diet; wks, weeks;WT, weight. 
All studies demonstrated accumulation of hepatic and/or tissue fat after HFD feeding. This may be due to an enhancement of lipogenic gene expression and decrement of lipolysis markers. Increased expression of lipogenic genes such as SREBP1c and its targets FAS and ACC were found in HFD mice. ${ }^{27,28,32}$ SREBP1c upregulated lipogenesis pathway by activating cholesterol synthesis (3-hydroxy3-methylglutarylcoenzymeA reductase (HMGCR)), fatty acid synthesis (ACC and FAS) and TG synthesis(glycerophosphate acyl transferase). SREBP1c downregulated lipolysis markers, Carnitine Palmitoyl Transferase-1(CPT-1) and peroxisome proliferator activated receptor- $\alpha$ (PPAR $\alpha)$, as well..$^{39}$ Lai et al., ${ }^{32}$ observed a significant reduction of SREBP1c, ACC, FAS and HMGCR along with elevated level of CPT-1and PPAR $\alpha$ after GEO and DADS consumption. In this study, GEO and DADS decreased lipid accumulation in the liver dose dependently by controlling lipid metabolism in longterm HFD-fed mice. Similarly, Xiao et al., ${ }^{27}$ reported low level of expression of SREBP1c along with increased adiponectin level after administration of SAMC. Han et al., ${ }^{33}$ measured the effect of ajoene on the expression of Liver X Receptor- $\alpha(\mathrm{LXR} \alpha)$ and ajoene effectively antagonized LXR $\alpha$ gene expression. LXR $\alpha$ is critical for transcription of SREBP- $1 \mathrm{c} .{ }^{40}$ According to Han et al., ${ }^{33}$ the attenuation of LXR $\alpha$ gene expression further supported by the suppression of lipogenic genes SREBP-1c, ACC, FAS and Stearoyl CoA Desaturase1(SCD-1). Furthermore, phosphorylation of Adenine Monophosphate Activated protein kinase(AMPK) was increased by garlic treatment in hepatocytes with a comparable decrease in S6 Kinase 1(S6K1) activity. ${ }^{33}$ AMPK inhibits phosphorylation of LXR $\alpha$ whereas S6K1 activates the phosphorylation of LXR $\alpha$ post-translationally. ${ }^{41}$

Fat accumulation in the liver results in peroxidation in hepatic tissues. lipid peroxidation is one of the possible mechanisms for the development NAFLD. ${ }^{42}$ Lipid peroxidation brings liver lesion through the formation of reactive metabolites. These reactive metabolites can directly toxify liver or indirectly trigger immune reactions..$^{27,33}$ This possibly bring the elevation of markers of liver injury as well as other metabolic biochemistry. Lai et al., ${ }^{32}$ demonstrated an elevation of AST by 2 fold and ALT by 5.5 fold after HFD feeding. Shin et al., ${ }^{29}$ ElDin et al., ${ }^{31}$ and Arhan et al., ${ }^{30}$ observed up-regulated levels AST and ALT in HFD mice and rabbits respectively. Further, Arhan et al., ${ }^{30}$ ElDin et al., ${ }^{31}$ and Lai et al. ${ }^{32}$ have shown an impaired levels of plasma glucose, FFA, TC, TG and insulin compared to the control group. Administration of various garlic extracts significantly diminished these analyses. Lai et al., ${ }^{32}$ indicated a dose dependent decrement of ALT, AST, TC and TG following treatment with SAMC. Similar result was reported by Arhan et al., ${ }^{30}$ and Shin et al., ${ }^{29}$ in garlic treated rabbits and mice respectively. El-Din et al., ${ }^{31}$ observed similar effect of garlic too.

Evidences indicated that hepatic lipid accumulation could induce immune reaction. ${ }^{27,33}$ Excessive accumulation of fat results in toxicity of the liver, leading to hepatocyte inflammation by inducing oxidative stress, infiltration of pro-inflammatory cytokines and hepatocyte mitochondrial dysfunction. ${ }^{43}$ An increased level of oxidative stress markers(Cytochrome P450 2E1(CYP2E1)), pro-inflammatory mediators(TNF-a,IL-1 圆, inducible nitric oxide synthase(iNOS)), cyclooxygenase-2 and chemokines (Monocyte Chemoattractant Protein-1, Macrophage Inflammatory Protein) were observed in HFD rats..$^{27,28,32}$ On the other hand, protective markers including antioxidative stress markers(catalase and GSH, GRx, GPx, GSOD and GST) $)^{27}$ and autophagic markers ${ }^{28}$ were significantly suppressed by HFD feeding. Lai et al., ${ }^{32}$ administered GEO and DADS and observed a dose dependent decrement of pro-inflammatory cytokine(IL-1 $\beta$, IL- 6 and TNF- $\alpha$, ) in the liver along with diminished oxidative stress markers,CYP2E1 and MDA. On the other hand, GEO and DADS supplementation upregulated GSH and other antioxidant enzymes (catalase, GPx, GRx, GST and GSOD). Similar finding was reported by Han YC et al., ${ }^{33}$ and Xiao et al., ${ }^{27}$ after delivery of ajoene and SAMC, respectively for HFD induced mice. In a similar fashion, El-Din et al., ${ }^{31}$ observed the restoration of the levels of antioxidative enzymes after garlic and/or onion treatment. More importantly, the elevated levels of phosphorylation of Mitogen Activated Protein Kinase(MAPK) and c-junk terminal kinase(JNK), but a decrease in the levels of phosphorylation of MEK/ERK1/2 were found in HFD induced NAFLD rat liver tissue. MAPK kinase signaling pathway including MAPK, JNK and MEK/ERK, plays important roles in insulin and inflammatory responses. Xiao et al., ${ }^{27}$ reported, the antagonist roles of treatment of SAMC against all the effects of HFD induced NAFLD on the phosphorylation of these kinases without influencing the baseline expression levels.

Garlic supplementation was found to be effective in diminishing apoptotic factors. Xiao et al., ${ }^{28}$ reported, the elevated levels of both intrinsic and extrinsic apoptotic factors in HFD induced NAFLD rats. On the contrary, the antiapoptoticBcl-2 families (Bcl-2 and Bcl-XL) were downregulated in HFD induced rats. In addition, the development of NAFLD in rats prohibited the phosphorylation of liver kinase B1 (LKB1), AMPK, phosphoinositide 3-kinase (PI3 K) and protein kinase $\mathrm{B} / \mathrm{Akt}$. However, treatment with $\mathrm{SAMC}$ restored the levels of the phosphorylation of LKB1, AMPK, PI3K and Akt, which leads to reduced caspase- 3 activity and apoptosis in the liver. In addition, treatment with SAMC was found to be effective in enhancing the hepatic autophagy through the prohibition of mTOR, contributing to the hepato-protective effects of SAMC. ${ }^{28}$

\section{Conclusion}

The primary objective of this review was to explore the therapeutic benefits of garlic in NAFLD by observing the levels of enzymes and liver histology. Since garlic is a common food supplement around the world, we believe that it is good to consider garlic or its derivatives as one of the therapeutic measures in the treatment strategy of NAFLD. The delivery of HFD to models brought liver injury. Co-administration of garlic antagonized this HFD induced liver damage by restricting the deposition of fat. Garlic supplements decreased the serum level of liver enzymes. In conclusion, garlic exerts its hepato-protective effect on various mechanisms involved in hepatic injury. Garlic suppressed inflammatory cytokines, lipogenic genes' expression, oxidative stress and apoptotic markers. On the other hand, its administration enhanced the expression of hepato-protective markers such as anti-inflammatory proteins, antioxidative enzymes and autophagy.

\section{Acknowledgments}

None.

\section{Conflicts of interest}

Authors declare that there is no conflict of interest.

\section{References}

1. Bedogni G, Miglioli L, Masutti F, et al. Prevalence of and risk factors for nonalcoholic fatty liver disease: the Dionysos nutrition and liver study. Hepatology. 2005;42(1):44-52. 
2. Matteoni CA, Younossi ZM, Gramlich T, et al. Nonalcoholic fatty liver disease: a spectrum of clinical and pathological severity. Gastroenterology. 1999;116(6):1413-1419.

3. Cairns S, Peters T. Biochemical analysis of hepatic lipid in alcoholic and diabetic and control subjects. Clinical Science. 1983;65(6):645652 .

4. Liver EAftSot, Diabetes EAftSo. EASL-EASD-EASO Clinical Practice Guidelines for the management of non-alcoholic fatty liver disease. Obesity Facts. 2016;9(2):65-90.

5. Ong JP, Younossi ZM. Epidemiology and natural history of NAFLD and NASH. Clinics in Liver Disease. 2007;11(1):1-16.

6. Tilg H, Moschen AR. Evolution of inflammation in nonalcoholic fatty liver disease: the multiple parallel hits hypothesis. Hepatology. 2010;52(5):1836-1846.

7. Saadeh S, Younossi ZM, Remer EM, et al. The utility of radiological imaging in nonalcoholic fatty liver disease. Gastroenterology. 2002;123(3):745-750

8. Marra F, Gastaldelli A, Baroni GS, et al. Molecular basis and mechanisms of progression of non-alcoholic steatohepatitis. Trends in Molecular Medicine. 2008;14(2):72-81.

9. Meshkani R, Adeli K. Hepatic insulin resistance, metabolic syndrome and cardiovascular disease. Clinical Biochemistry. 2009;42(13):13311346.

10. Wahrenberg H, Lönnqvist F, Arner P. Mechanisms underlying regional differences in lipolysis in human adipose tissue. Journal of Clinical Investigation. 1989;84(2):458.

11. Musso G, Gambino R, Cassader M, et al. Meta-analysis: natural history of non-alcoholic fatty liver disease (NAFLD) and diagnostic accuracy of non-invasive tests for liver disease severity. Annals of Medicine. 2011;43(8):617-649

12. Fan Y, Bergmann A. Apoptosis-induced compensatory proliferation The Cell is dead. Long live the Cell! Trends in Cell Biology. 2008;18(10):467-473.

13. Feldstein AE, Canbay A, Angulo P, et al. Hepatocyte apoptosis and fas expression are prominent features of human nonalcoholic steatohepatitis. Gastroenterology. 2003;125(2):437-443.

14. Feldstein AE, Wieckowska A, Lopez AR, et al. Cytokeratin-18 fragment levels as noninvasive biomarkers for nonalcoholic steatohepatitis: a multicenter validation study. Hepatology. 2009;50(4):1072-1078.

15. Lazaridis N, Tsochatzis E. Current and future treatment options in nonalcoholic steatohepatitis (NASH). Expert Review of Gastroenterology \& Hepatology. 2017;11(4):357-369.

16. Ofosu A, Ramaia D, Reddy M. Non-alcoholic fatty liver disease: controlling an emerging epidemic, challenges, and future directions. Annals of Gastroenterology. 2018;31(3):1-8.

17. Lewis JD, Ferrara A, Peng T, et al. Risk of bladder cancer among diabetic patients treated with pioglitazone: interim report of a longitudinal cohort study. Diabetes Care. 2011;34(4):916-922.

18. Gee PT. Unleashing the untold and misunderstood observations on vitamin E. Genes \& Nutrition. 2011;6(1):5.

19. Amagase $\mathrm{H}$, Petesch BL, Matsuura $\mathrm{H}$, et al. Intake of garlic and its bioactive components. The Journal of Nutrition. 2001;131(3):955S-962S.

20. Amagase H. Clarifying the real bioactive constituents of garlic. The Journal of Nutrition. 2006;136(3):716S-725S.

21. Mohamed MS, Abdel-Kader MM, Kassem SS. Effect of dietary garlic and onion on liver and tibial mineral concentrations in omega-3 fatty acids rich oil fed rats. Agric Biol JN Am. 2011;2(5):745-751.

22. Mohammadi A, Oshaghi EA. Effect of garlic on lipid profile and expression of LXR alpha in intestine and liver of hypercholesterolemic mice. Journal of Diabetes \& Metabolic Disorders. 2014;13(1):20.

23. Fujisawa H, Watanabe K, Suma K, et al. Antibacterial potential of garlic-derived allicin and its cancellation by sulfhydryl compounds. Bioscience, Biotechnology, and Biochemistry. 2009;73(9):1948-1955.

24. Moher D, Liberati A, Tetzlaff J, et al. Preferred reporting items for systematic reviews and meta-analyses: the PRISMA statement. PLoS Medicine. 2009;6(7):e1000097.

25. Vries R, Hooijmans CR, Langendam MW, et al. A protocol format for the preparation, registration and publication of systematic reviews of animal intervention studies. Evidence-based Preclinical Medicine. 2015;2(1):1-9.

26. Hooijmans CR, Rovers MM, de Vries RB, et al. SYRCLE's risk of bias tool for animal studies. BMC Medical Research Methodology. 2014;14(1):43

27. Xiao J,Ching YP,LiongEC, etal.Garlic-derivedS-allylmercaptocysteine is a hepato-protective agent in non-alcoholic fatty liver disease in vivo animal model. European Journal of Nutrition. 2013;52(1):179-191.

28. Xiao J, Guo R, Fung ML, et al. Garlic-derived S-allylmercaptocysteine ameliorates nonalcoholic fatty liver disease in a rat model through inhibition of apoptosis and enhancing autophagy. Evidence-Based Complementary and Alternative Medicine. 2013;2013:642920.

29. Shin JH, Lee CW, Oh SJ, et al. Hepatoprotective effect of aged black garlic extract in rodents. Toxicological Research. 2014;30(1):49.

30. Arhan M, Öztürk HS, Turhan N, et al. Hepatic oxidant/antioxidant status in cholesterol-fed rabbits: Effects of garlic extract. Hepatology Research. 2009;39(1):70-77.

31. El-Din HS, Sabra ANA, Hammam OA. Pharmacological and Antioxidant Actions of Garlic and - Or Onion in Non Alcoholic Fatty Liver Disease (Nafld) in Rats. Journal of the Egyptian Society of Parasitology. 2014;44(2)

32. Lai YS, Chen WC, Ho CT, et al. Garlic essential oil protects against obesity-triggered nonalcoholic fatty liver disease through modulation of lipid metabolism and oxidative stress. Journal of Agricultural and Food Chemistry. 2014;62(25):5897-5906.

33. Han CY, Ki SH, Kim YW, et al. Ajoene, a stable garlic by-product, inhibits high fat diet-induced hepatic steatosis and oxidative injury through LKB1-dependent AMPK activation. Antioxidants \& Redox Signaling. 2011;14(2):187-202.

34. Qamar A, Siddiqui A, Kumar H. Fresh garlic amelioration of high-fatdiet induced fatty liver in albino rats. Journal of the Pakistan Medical Association. 2015;65(10):1102-1107.

35. Chalasani N, Younossi Z, Lavine JE, et al. The diagnosis and management of non-alcoholic fatty liver disease: practice guideline by the American Gastroenterological Association, American Association for the Study of Liver Diseases, and American College of Gastroenterology. Gastroenterology. 2012;142(7):1592-1609.

36. Wang RT, Koretz RL, Yee HF. Is weight reduction an effective therapy for nonalcoholic fatty liver?: A systematic review. The American Journal of Medicine. 2003;115(7):554-559.

37. Boettcher E, Csako G, Pucino F, et al. Meta-analysis: pioglitazone improves liver histology and fibrosis in patients with non-alcoholic steatohepatitis. Alimentary Pharmacology \& Therapeutics. 2012;35(1):66-75. 
38. Colmers IN, Bowker SL, Majumdar SR, et al. Use of thiazolidinediones and the risk of bladder cancer among people with type 2 diabetes: a metaanalysis. Canadian Medical Association Journal. 2012;184(12):E675E83.

39. Pan MH, Lai CS, Tsai ML, et al. Chemoprevention of nonalcoholic fatty liver disease by dietary natural compounds. Molecular Nutrition \& Food Research. 2014;58(1):147-171.

40. Repa JJ, Liang G, Ou J, et al. Regulation of mouse sterol regulatory element-binding protein-1c gene (SREBP-1c) by oxysterol receptors, LXR $\alpha$ and LXR $\beta$. Genes \& Development. 2000;14(22):2819-2830.

41. Hwahng SH, Ki SH, Bae EJ, et al. Role of adenosine monophosphate- activated protein kinase-p70 ribosomal S6 kinase-1 pathway in repression of liver $\mathrm{X}$ receptor-alpha-dependent lipogenic gene induction and hepatic steatosis by a novel class of dithiolethiones. Hepatology. 2009;49(6):1913-1925.

42. Reddy JK, Hashimoto T. Peroxisomal $\beta$-oxidation and peroxisome proliferator-activated receptor $\alpha$ : an adaptive metabolic system. Annual Review of Nutrition. 2001;21(1):193-230.

43. Fromenty B, Robin M, Igoudjil A, et al. The ins and outs of mitochondrial dysfunction in NASH. Diabetes \& Metabolism. 2004;30(2):121-138. 